\title{
A Broadband Variable Passive True Time Delay at 2-12GHz
}

\author{
Li Wenyuan ${ }^{+}$, Cao Mingxi \\ Institute of RF-\&OE-ICs, Southeast University, Nanjing 210096, China
}

\begin{abstract}
A fully integrated passive True Time Delay (TTD) with 66ps continuous changing delay time and less than $11 \%$ delay variation has been realized in a TSMC 0.18 um CMOS technology. A six-stage trombone configuration is employed to provide $55 \mathrm{ps}$ tuning capability with $11 \mathrm{ps}$ delay resolution for the coarse tuning part. The fine tuning part employs six LC delay cells with varactor added in both ground branches to provide $11 \mathrm{ps}$ continuous delay time. The simulated input and output return loss is better than $-10 \mathrm{~dB}$ over the $2-12 \mathrm{GHz}$ range and the insertion loss is $-23 \pm 1.5 \mathrm{~dB}$ at $10 \mathrm{GHz}$. The simulated worst-case IIP3 at $6 \mathrm{GHz}$ is $1.28 \mathrm{dBm}$ and the proposed TTD core occupies $1.782 \mathrm{~mm}^{2}$, respectively.
\end{abstract}

Keywords: passive, True Time Delay (TTD), CMOS, trombone configuration.

\section{Introduction}

True time delay (TTD) circuit is a critical block in pulse-based radars and broadband beam forming transceivers which provide flat delay time over a large frequency range [1]. Over the past few decades, various passive and active delay techniques have been explored extensively. MEMS based TTD [2] and Photonic TTD module [3] exhibit large total variable delay, low insertion loss and low delay variation. However, in low cost and integrated systems, these methods are not appropriate. With regard to integrated TTD methods, the RC all-pass filter can offer large variable delay time with only a small chip area [4,5]. However, it is only researched for low frequencies so far. In this paper, the proposed TTD will employ a new structure, which aims to acquire a flat delay response over $2-12 \mathrm{GHz}$ with max $11 \%$ delay variation.

\section{Architecture}
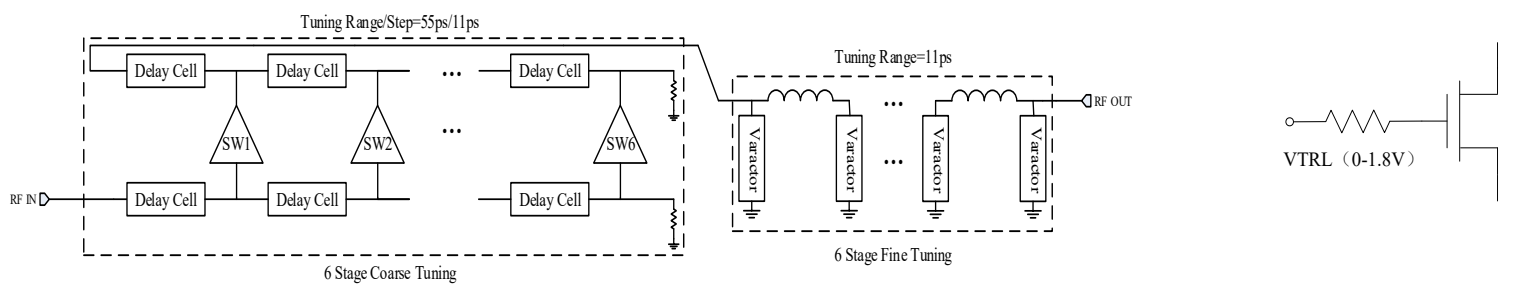

Fig. 1: Block diagram of the proposed TTD circuit

Fig. 2: Implementation of NMOS switches

Fig. 1 shows the basic structure of the proposed TTD circuit. In order to achieve the high tuning resolution and large total variable delay, Coarse tuning part and fine tuning part are combined together [6], which is aimed to achieve $66 \mathrm{ps}$ continuous changing delay time over the $2-12 \mathrm{GHz}$ range. With six active switches (SW1-SW6) distributed along the input and output delay lines [7], the coarse tuning part provides 55ps delay time with five discrete delay tuning steps of $11 \mathrm{ps}$, respectively. What's more, the fine tuning part, based on varactor mechanisim, provides $11 \mathrm{ps}$ continuous variable delay time in total so as to cover the $11 \mathrm{ps}$ step of the

\footnotetext{
${ }^{+}$Corresponding author. Tel.: + 86-258-3793303-8109; fax: +86-25-63792882.

E-mail address:1wy555@seu.edu.cn.
} 
adjacent delay cell of the coarse tuning part. The active switches in this design are implemented with NMOS devices with large series resistor connected to gate to prevent signal leakage shown in Fig.2.

\section{Building Block}

\subsection{Design of the Coarse Tuning Part}

The delay cell of the proposed coarse tuning part is shown in Fig. 3a. By cascading multistage delay cells together as shown in Fig. 3b, larger delay time can be obtained. When the cutoff frequency is far greater than the operating frequency, the characteristic impedance $Z_{0}$ and the group delay $\tau$ of the delay cell are:

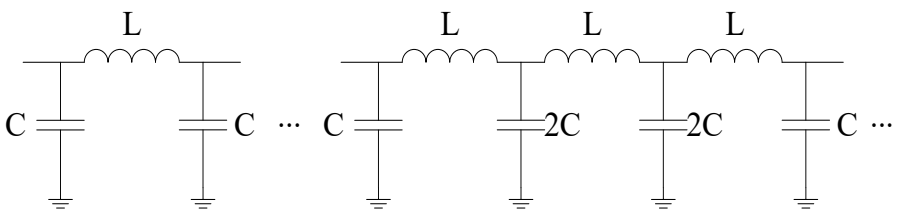

Fig. 3: (a) Delay cell

(b) Cascading delay cell

$$
\begin{aligned}
& \mathrm{Z}_{0}=\sqrt{\mathrm{L} / 2 \mathrm{C}} \\
& \tau=\sqrt{2 \mathrm{LC}}
\end{aligned}
$$

The cutoff angular frequency of each delay cell is:

$$
\mathrm{W}_{\mathrm{c}}=\sqrt{\mathrm{RL}^{2 \mathrm{~L}}}
$$

Fig. 4 illustrates the delay response of $\mathrm{LC}$ delay cell from $1 \mathrm{~Hz}$ to $30 \mathrm{GHz}$, It can be observed that $\mathrm{W}_{\mathrm{c}}$ drops when larger delay time is realized in each delay cell and lead to a larger delay variation at high frequencies. As less delay time in each delay cell contributes to minimizing the delay variation, the proposed TTD circuit adopts a multistage architecture. Each delay cell only provides $1 / \mathrm{N}$ of the total delay time by cascading $\mathrm{N}$-stage delay cells. Through this solution, each delay cell's cutoff frequency will be enhanced greatly, and hence, enables a low delay variation at high frequency. However, the more delay stages used in the design, the larger chip area will be employed in practice and hence $\mathrm{L}$ is designed to be $225 \mathrm{pH}$, and the capacitance $\mathrm{C}$ is $45 \mathrm{fF}$.

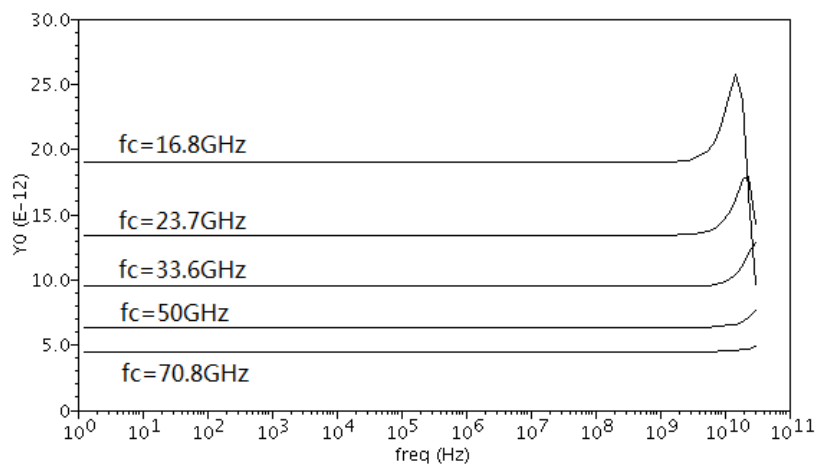

Fig. 4: Delay response of LC delay cell with different cutoff frequencies $f_{c}$

\subsection{Design of the Fine Tuning Part}

Fig. 5 shows the design of six-stage LC fine tuning part. In order to relax the impedance matching between coarse tuning part and fine tuning part, the fine tuning part is needed to have a low characteristic impedance since the coarse Tuning part has a low output impedance. This lead to a large varactor $\mathrm{C}_{\mathrm{var}}$ and a small inductance L. However, a too large varactor may result in two problems. First, a too large varactor results in large amplitude variation during the fine delay tuning for the insertion loss of the varactor varies as its capacitance changes. What's more, it degrades the fine delay tuning ability because of large parasitic capacitance introduced by the varactor. As a result, $\mathrm{L}$ is designed to be $225 \mathrm{pH}$ and the varactor ranges from $44 \mathrm{fF}$ to $95 \mathrm{fF}$ shown in Fig.6. It is worth noting that these parameters in each stage may change a little during the layout implementation because of all kinds of parasitic. 


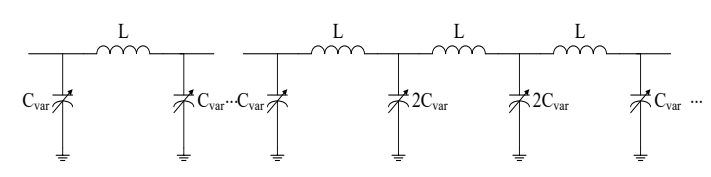

Fig. 5: Design of the fine tuning part

\section{Layout and Simulation Results}

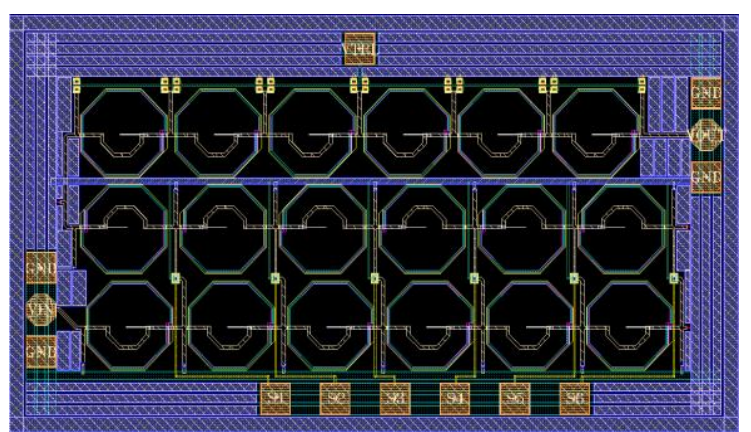

Fig. 7: Layout of the TTD

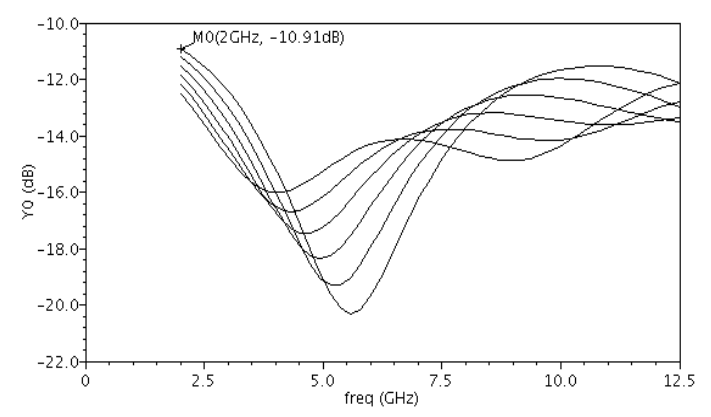

Fig. 9: S22 of the TTD in six states

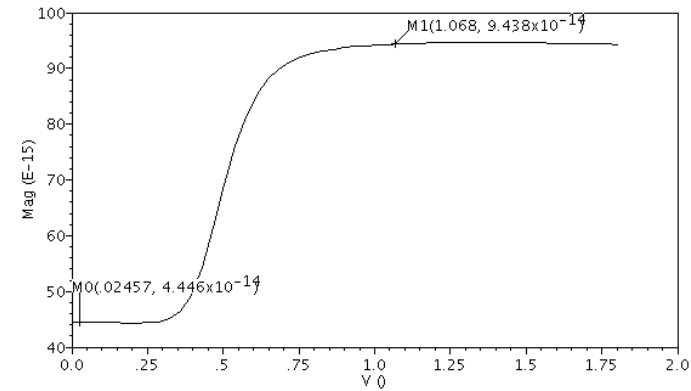

Fig. 6: Varactor characteristic

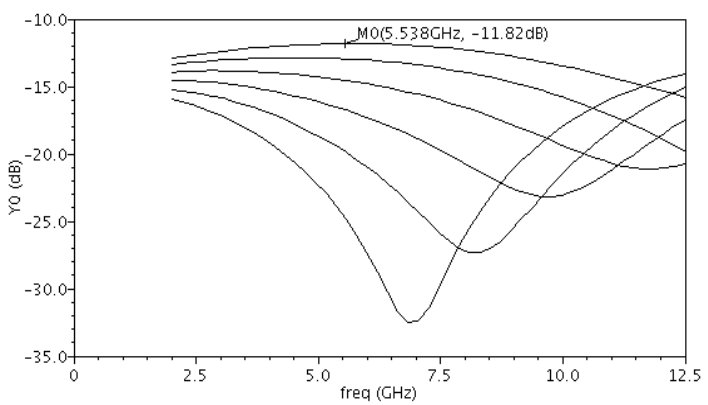

Fig. 8: S11 of the TTD in six states

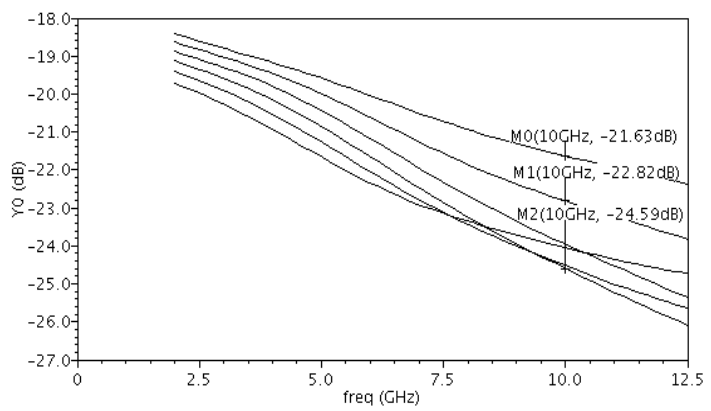

Fig. 10: S21 of the TTD in six states

The fully integrated passive TTD has been realized in a TSMC 0.18um CMOS technology. Fig. 7 shows the layout of the proposed TTD and the chip area is $1.782 \mathrm{~mm}^{2}$. The simulated S parameter are shown in Fig. 8, Fig. 9 and Fig. 10. The S11 and S22 are well below $-10 \mathrm{~dB}$ across the whole simulation frequency of 2-12GHz. The insertion loss of the proposed TTD is $-23 \pm 1.5 \mathrm{~dB}$ at $10 \mathrm{GHz}$.

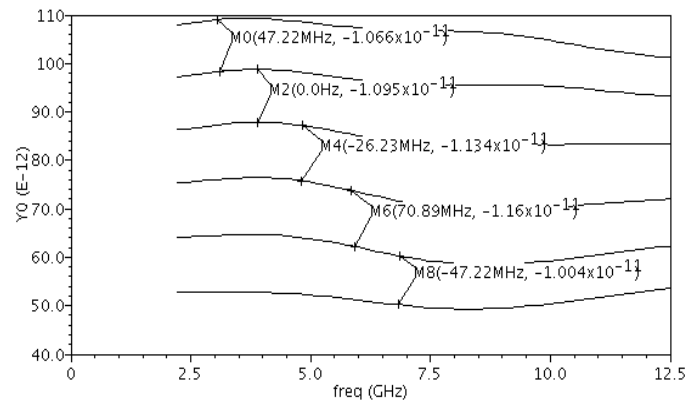

Fig. 11: Group delay of the TTD in six states

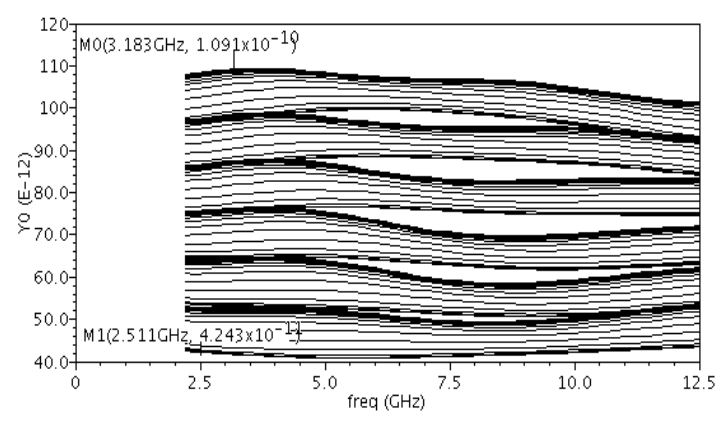

Fig. 12: Group delay of the TTD in all states

Fig. 11 shows the simulated group delay of six states which characterizes how much signal distortion is caused by the TTD. It can be seen that the total achieved changing delay time is 66ps. The group delay of all simulated states is shown in Fig. 12. For phased array antennas, the beam direction is controlled by the relative delay difference between adjacent antennas. The relative phase delay that can be provided by the proposed TTD is shown Fig. 13. 


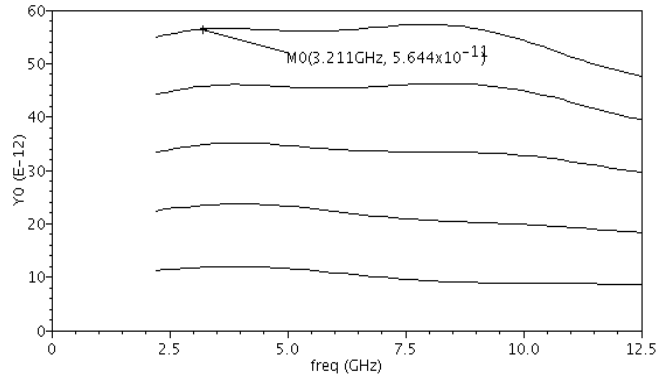

Fig. 13: Relative delay of the TTD in six states

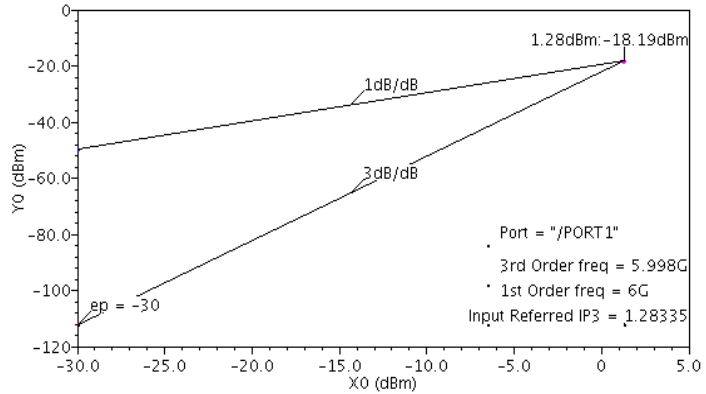

Fig. 14: Worst-case IIP3@6GHz

The simulated worst-case IIP3 is $1.28 \mathrm{dBm}$ at $6 \mathrm{GHz}$ shown in Fig. 14. Fig. 15 and Fig. 16 gives the simulated time domain delay response. It can be seen that time domain delay response is almost same as group delay. Fig. 17 shows the delay variation of different delay settings which expressed in percentage of the total variable delay time so as to evaluate the flatness of the achieved delay response:

$$
\Delta \tau(\%)=\frac{\max (\tau)-\min (\tau)}{\tau_{\text {tot }}} \times 100 \%
$$

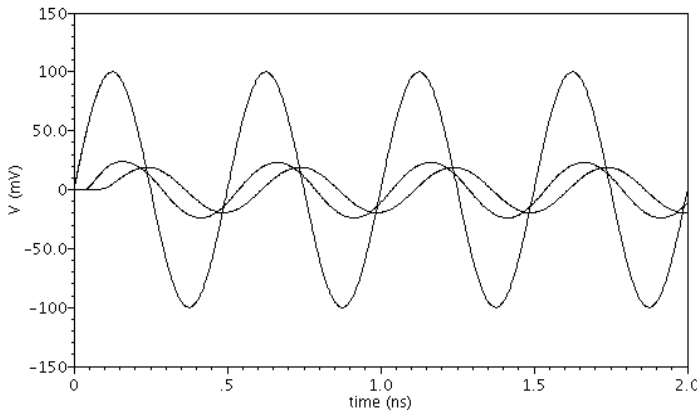

Fig. 15: Time domain delay response@2 $2 \mathrm{GHz}$

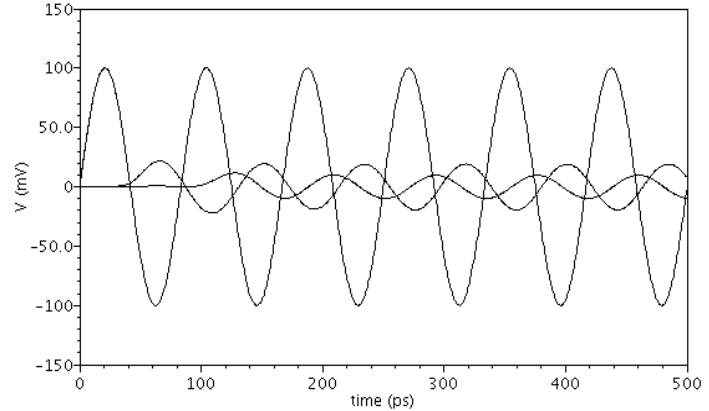

Fig. 16: Time domain delay response@12GHz

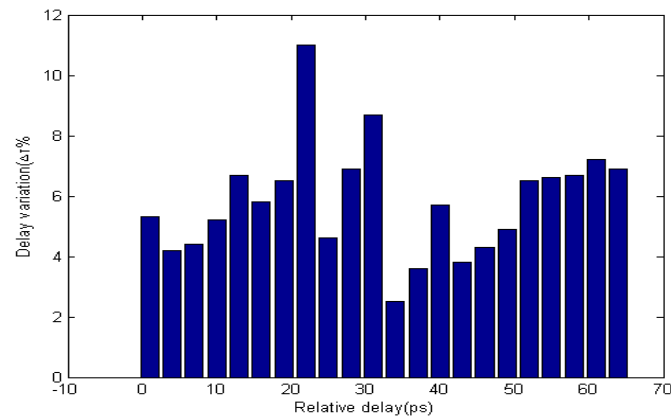

Fig. 17: Delay variation of different delay settings

Table 1: Performance summary of the TTD

\begin{tabular}{|c|c|c|}
\hline Reference & Simulation & EM \\
\hline Max delay(ps) & 66 & 66 \\
\hline Bandwidth $(\mathrm{GHz})$ & $2-12$ & $2-12$ \\
\hline Resolution & continuous & continuous \\
\hline Delay variation & $<11 \%$ & $<11 \%$ \\
\hline S11(dB) & $<-10$ & $<-10$ \\
\hline S22(dB) & $<-10$ & $<-10$ \\
\hline S21@10GHz(dB) & $-23 \pm 1.5$ & $-24 \pm 1.5$ \\
\hline IIP3@6GHz $(\mathrm{dBm})$ & 1.28 & 1.28 \\
\hline Core area $\left(\mathrm{mm}^{2}\right)$ & 1.782 & 1.782 \\
\hline
\end{tabular}

The increasing complexity and operating frequency of a modern microwave circuit make the parasitic effects of interconnects very critical in layout design. Several modeling methods for an interconnect have been introduced. In this paper, the electromagnetic (EM) based approach is employed which is derived from the 
numerical solutions of Maxwell's equations that describe the EM behaviors of the physical structures in advance without fabrication at high frequencies. Table I shows the simulated and EM performance of the proposed TTD. It can be seen that the simulated results almost consist with the EM results.

\section{Conclusion}

A passive broadband TTD circuit with 66ps continuous variable delay time is demonstrated in a TSMC 0.18um CMOS technology. With the proposed matching technique, a flat delay response is achieved from $2 \mathrm{GHz}$ to $12 \mathrm{GHz}$ with less than $11 \%$ delay variation.

\section{Acknowledgements}

The authors would like to acknowledge the support from the Project supported by the Natural Science Foundation of China (No.61471119), the Priority Academic Program Development of Jiangsu Higher Education Institutions (PAPD), and Topnotch Academic Programs Project of Jiangsu Higher Education Institutions (TAPP) PPZY2015A035.

\section{References}

[1] Ma Q, Leenaerts D, Mahmoudi R. A 10-50GHz True-Time-Delay phase shifter with max 3.9\% delay variation[C]. IEEE Radio Frequency Integrated Circuits Symposium. IEEE, 2014: 83-86.

[2] Kim M, Hacker J B, Mihailovich R E, et al. A DC-to-40 GHz four-bit RF MEMS true-time delay network[J]. IEEE Microwave and Wireless Components Letters, 2001, 11(2): 56-58.

[3] Chen Y, Chen R T. A fully packaged true time delay module for a K-band phased array antenna system demonstration [J]. IEEE Photonics Technology Letters, 2002, 14(8): 1175-1177.

[4] Garakoui S K, Klumperink E A M, Nauta B, et al. A 1-to-2.5 GHz phased-array IC based on g m-RC all-pass time-delay cells[C]. IEEE International Solid-State Circuits Conference. IEEE, 2012: 80-82.

[5] Ulusoy A C, Schleicher B, Schumacher H. A tunable differential all-pass filter for UWB true time delay and phase shift applications[J]. IEEE Microwave and Wireless Components Letters, 2011, 21(9): 462-464.

[6] Ma Q, Leenaerts D, Mahmoudi R. A 12ps true-time-delay phase shifter with 6.6\% delay variation at 2040GHz[C]. IEEE Radio Frequency Integrated Circuits Symposium (RFIC). IEEE, 2013: 61-64.

[7] Park S, Jeon S. A 15-40 GHz CMOS true-time delay circuit for UWB multi-antenna systems[J]. IEEE Microwave and Wireless Components Letters, 2013, 23(3): 149-151. 